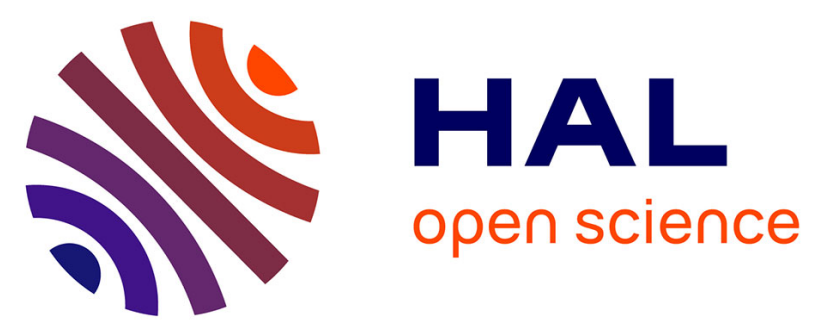

\title{
Structural origin of the drastic modification of second harmonic generation intensity pattern occurring in tail muscles of climax stages xenopus tadpoles.
} Gaëlle Recher, Pascal Coumailleau, Denis Rouède, François Tiaho

\section{- To cite this version:}

Gaëlle Recher, Pascal Coumailleau, Denis Rouède, François Tiaho. Structural origin of the drastic modification of second harmonic generation intensity pattern occurring in tail muscles of climax stages xenopus tadpoles.. Journal of Structural Biology, 2015, 190 (1), pp.1-10. 10.1016/j.jsb.2015.03.003 . hal-01134174

HAL Id: hal-01134174 https://hal-univ-rennes1.archives-ouvertes.fr/hal-01134174

Submitted on 4 May 2015

HAL is a multi-disciplinary open access archive for the deposit and dissemination of scientific research documents, whether they are published or not. The documents may come from teaching and research institutions in France or abroad, or from public or private research centers.
L'archive ouverte pluridisciplinaire HAL, est destinée au dépôt et à la diffusion de documents scientifiques de niveau recherche, publiés ou non, émanant des établissements d'enseignement et de recherche français ou étrangers, des laboratoires publics ou privés. 


\title{
Structural origin of the drastic modification of second harmonic generation intensity pattern occurring in tail muscles of climax stages xenopus tadpoles
}

\author{
Gaëlle Recher $^{\mathrm{a}, 1,2}$, Pascal Coumailleau ${ }^{\mathrm{a}, 3}$, Denis Rouède $^{\mathrm{b}}$ and François Tiaho ${ }^{\mathrm{a}, 3}$
}

${ }^{a}$ UMR CNRS 6026, Université de Rennes1, Campus de Beaulieu, Rennes, F-35000, France.

bIPR, CNRS, UMR-CNRS UR1- 6251, Université de Rennes1, Campus de Beaulieu, Rennes, F-35000, France

${ }^{1}$ Wellcome Trust/Cancer Research UK Gurdon Institute, University of Cambridge, Tennis Court Road, Cambridge CB2 1QR, UK

${ }^{2}$ Department of Physiology, Development and Neuroscience, University of Cambridge, Downing Street, Cambridge CB2 3DY, UK

${ }^{3}$ IRSET, INSERM, U1085, Université de Rennes1, Campus de Beaulieu, Rennes, F-35000, France.

Corresponding authors:

- E-mail: francois.tiaho@univ-rennes1.fr

- Phone : 33223235133

- Postal address : Bâtiment 13, campus de Beaulieu, 263 avenue du général Leclerc, 35042

Rennes Cedex, France 


\begin{abstract}
Second harmonic generation (SHG) microscopy is a powerful tool for studying submicron architecture of muscles tissues. Using this technique, we show that the canonical single frequency sarcomeric SHG intensity pattern (SHG-IP) of premetamorphic xenopus tadpole tail muscles is converted to double frequency (2f) sarcomeric SHG-IP in metamorphic climax stages due to massive physiological muscle proteolysis. This conversion was found to rise from $7 \%$ in premetamorphic muscles to about $97 \%$ in fragmented muscular apoptotic bodies. Moreover a $66 \%$ conversion was also found in non-fragmented metamorphic tail muscles. Also, a strong correlation between predominant $2 \mathrm{f}$ sarcomeric SHG-IPs and myofibrillar misalignment is established with electron microscopy. Experimental and theoretical results demonstrate the higher sensitivity and the supra resolution power of SHG microscopy over TPEF to reveal 3D myofibrillar misalignment. From this study, we suggest that $2 \mathrm{f}$ sarcomeric SHG-IP could be used as signature of triad defect and disruption of excitation-contraction coupling. As the mechanism of muscle proteolysis is similar to that found in mdx mouse muscles, we further suggest that xenopus tadpole tail resorption at climax stages could be used as an alternative or complementary model of Duchene muscular dystrophy.
\end{abstract}

Key words: skeletal muscle, second harmonic generation microscopy, two photon excitation fluorescence, electron microscopy, xenopus, apoptosis.

\title{
1. Introduction
}

Excitation-contraction coupling at sarcomeric level required triads composed of a central transverse tubule (T-tubule), flanked by two closely apposed sarcoplasmic reticulum (SR) terminal cisternae. Triads and myofibrils are regularly ordered in adult skeletal muscles and this organization is developmentally regulated (Ezerman and Ishikawa, 1967; Veratti, 1961). Structural intimate relation between the two has been first observed at Z-bands of amphibian larvae tail muscles (Porter and Palade, 1957), suggesting that the functional efficiency of triads could be coupled to perfect structural myofibrillar alignment at their A-, M-, I- and Zbands. This perfect myofibrillar arrangement is altered during muscle development, damage and regeneration (Takekura et al., 2001; Yeung et al., 2002). Since myofibrillar misalignment could damage triads and disrupt excitation-contraction coupling, a non-invasive label-free method that can probe in vivo or in vitro the $3 \mathrm{D}$ muscular micrometric architecture in thick muscle tissues is therefore expected to provide paramount structural information concerning local proteolysis and alteration occurring at triads.

Second harmonic generation (SHG) microscopy is a low-invasive technique that relies on a nonlinear optical interaction with hyperpolarizable endogeneous molecules like collagen and myosin causing scattered coherent radiation at twice the fundamental frequency (Campagnola et al., 2002). Hence, SHG highlights the myosin filaments of sarcomeric Abands. It has proved to be an extremely beneficial contrast mechanism for label-free imaging of skeletal muscle in vivo, in disease state as well as during dynamic contraction (Buttgereit et al., 2013; Llewellyn et al., 2008; Nucciotti et al., 2010; Plotnikov et al., 2008; Prent et al., 2008; Ralston et al., 2008; Rouede et al., 2013b; Rouede et al., 2014). Our research group has previously shown that the predominant feature of sarcomeric SHG intensity pattern (SHG-IP) is a single peak per sarcomere i.e. single frequency (1f) in normal physiological condition (Recher et al., 2009) and that this pattern is converted to two peaks per sarcomere i.e. double frequency (2f) upon proteolysis, mechanical and chemical stresses, laser induced photodamage and muscular dystrophy (Recher et al., 2011a; Recher et al., 2011b; Rouede et al., 2013b). More recently we have shown that these patterns originate from myofibrillar misalignment (Rouede et al., 2013a) and are characterized by triads alteration in mdx mouse 
model of Duchene muscular dystrophy (Rouede et al., 2014) suggesting that SHG microscopy could detect structural disruption of excitation-contraction coupling. However the mechanism underlying $2 \mathrm{f}$ sarcomeric SHG-IPs is still not completely elucidated. Several mechanisms have been proposed to explain the ultrastructural origin of this $2 \mathrm{f}$ sarcomeric SHG-IP encompassing centrosymmetry of antiparallel myosin tails arrangement at the M-band in normal cardiac muscles (Boulesteix et al., 2004; Garcia-Canadilla et al., 2014; Plotnikov et al., 2006), disease dependent myofibrillar deformation or change of myosin orientation (Friedrich et al., 2010), proteolysis induced intra-sarcomeric misalignment of myosin thick filaments (Rouede et al., 2011) and myofibrillar misalignment (Rouede et al., 2013a). Difficulties in correlating SHG and electron microscopy (EM) images represent the major technological limitation. If EM is the gold standard imaging technique when nanometric resolution is needed, its multiple-step processing of biological samples frequently causes alteration of tissue structure (Endo, 1964; Jayasinghe and Launikonis, 2013; Launikonis and Stephenson, 2002; Soeller and Cannell, 1999) and observations are often limited to ultra-thin slices (about $100 \mathrm{~nm}$ ) of muscle tissue. In these conditions, correlation between $2 \mathrm{f}$ sarcomeric SHG-IP and myofibrillar misalignment at EM level is only possible when $2 \mathrm{f}$ sarcomeric SHGIP is abundant. Based on our previous result showing that muscle proteolysis increases the percentage of $2 \mathrm{f}$ sarcomeric SHG-IP (Recher et al., 2011b; Recher et al., 2009; Rouede et al., 2013a; Rouede et al., 2011), we hypothesize that amphibian tadpole tail which undergoes physiological muscle proteolysis with multiple apoptotic pathways during spontaneous metamorphosis (Kerr et al., 1974), might be a good tissue source of $2 \mathrm{f}$ sarcomeric SHG-IPs enabling precise determination of their ultra-structural origin.

Amphibian metamorphosis is characterized by several physiological transformations occurring in a predetermined order 1) proliferation and differentiation of adult tissues triggered by thyroid hormone (TH) signaling and 2) delayed degeneration of many larval tissues (Ishizuya-Oka, 2011; Nakajima et al., 2012). Hence low levels of TH at prometamorphic stages induced hind limb morphogenesis while tail muscle cell death is delayed until metamorphic climax stages when high levels of TH are achieved. High TH level induced spontaneous cell death during tail involution of the anuran xenopus tadpole, is considered as a model for describing and defining cellular events of physiological program cell death (apoptosis) (Berry et al., 1998; Du Pasquier et al., 2006; Kerr et al., 1972; Kerr et al., 1974; Rowe et al., 2005; Thornberry, 1997). More recently it has been suggested that TH induced reactive oxygen species (ROS) dependent proteolysis is another pathway that plays an important role in amphibian tadpoles tail regression during metamorphosis (Inoue et al., 2004; Johnson et al., 2013; Kashiwagi et al., 1999; Menon and Rozman, 2007). Despite the general agreement that $\mathrm{TH}$ initiates the molecular program leading to apoptosis and tail resorption, the downstream molecular mechanisms and their spatio-temporal characteristic are still not elucidated. Interestingly, we have previously shown in premetamorphic xenopus tadpole that oxidative stress induces tail muscle proteolysis that is characterized by $2 \mathrm{f}$ sarcomeric SHG-IPs (Recher et al., 2011a) suggesting that the appearance of this pattern could be used to determine the contribution of ROS dependent proteolysis in climax stage metamorphosis. Altogether both caspase-dependent and ROS-dependent proteolysis processes that occur during xenopus tail muscle involution are supportive of our hypothesis that climax stage xenopus tail muscles might be a rich source of $2 \mathrm{f}$ sarcomeric SHG-IPs.

In order to gain insight into the ultrastructural mechanism responsible for $2 \mathrm{f}$ sarcomeric SHG-IPs occurring in tail muscles proteolysis during metamorphosis, we undertook SHG, TPEF and electron microscopy (EM) studies from premetamorphic, prometamorphic and metamorphic climax stage of xenopus tadpoles. 


\section{Experimental methods}

\subsection{Tissue preparation}

Nieuwkoop and Faber (NF) premetamorphic stage 50-53, prometamorphic stage 55-57 and metamorphic climax stage 60-63 Xenopus laevis tadpoles (national breeding facility of xenopus animals in Rennes, France) were anesthetized in MS222 $\left(0.5 \mathrm{mg} \cdot \mathrm{mL}^{-1}\right)$. At least 1015 tadpoles for each group were used. For SHG imaging, fresh tails were cut and mounted in an imaging chamber stabilized and sealed between two coverslips (POC System, Pecon), in Mark's Modified Ringer (MMR). For metamorphic climax stage tadpoles, only tissues from the distal third of their tails, characterized by advanced regression, were used for imaging.

For $\alpha$-actinin and myosin immunohistochemistry experiments, MS222-euthanized whole tadpoles were fixed at $4{ }^{\circ} \mathrm{C}$ overnight in MMR containing $4 \%$ paraformaldehyd (PFA) then rinsed at least three times with MMR. 10-15 $\mu \mathrm{m}$ cryostat sections of tail tissues, mounted on gelatin-coated coverslips, were permeabilized with buffer containing $0.5 \%$ triton and $5 \%$ fetal calf serum for 10 minutes. They were then incubated at $4{ }^{\circ} \mathrm{C}$ over night in MMR containing $0.1 \%$ triton, $5 \%$ fetal calf serum and one of the following primary antibody. Sarcomeric Zlines were targeted with $\alpha$-actinin antibody $(1: 100$, mouse monoclonal IgM, ab9465, Abcam, Cambridge, MA, USA) and A-bands were targeted with myosin antibody (1:60, MF20 mouse monoclonal IgG developed by D. A. Fischman or 1:50, F59 mouse monoclonal IgG developed by F.E. Stockdale, both obtained from the Developmental Studies Hybridoma Bank, University of Iowa). Slices were rinsed three times in MMR and incubated at $4{ }^{\circ} \mathrm{C}$ over night in Ringer containing $0.1 \%$ triton, $5 \%$ fetal calf serum and secondary antibody (1:100, Alexa Fluor 594 goat antimouse IgG, Molecular Probes, Eugene, OR, USA). After being rinsed three times, the tissue was covered with a drop of mounting medium (Vectashield, Vector Burlingame, CA) and a second coverslip was laid on. After being sealed, preparations were imaged.

For transmission electron microscopy (TEM), blocs of approximately $1 \mathrm{~mm}^{3}$ from PFAprefixed 3 premetamorphic stage 53 and 3 metamorphic climax stage 63 Xenopus laevis tadpoles tail muscles were cut and PBS-washed. The blocs were further fixed in glutaraldehyde $\left(2.5 \%, 4^{\circ} \mathrm{C}\right.$, in $0.2 \mathrm{M}$ cacodylate buffer, $\left.\mathrm{pH} 7.2\right)$ for one hour and washed in cacodylate buffer. The next steps of sample preparation leading to TEM imaging were previously reported (Rouède et al 2011). Briefly, blocs were post-fixed in osmium tetroxide $(2 \%)$ and dehydrated in a graded acetone series. They were impregnated firstly into a mix of Epon, Araldite and acetone and secondly into a mix of Epon, Araldite and DMP30 then laid down into molds and polymerized. After bloc desiccation, ultra fine slices were made with an ultra-microtome (Ultracut E, Reichert), mounted on a copper grid and stained with uranyl acetate $(2.5 \%)$. Slices were then dipped in lead citrate and finally rinsed. Imaging was performed using a JEOL 100CXII microscope at $80 \mathrm{kV}$ accelerating voltage, at the TEM mRIC platform (http://microscopie.univ-rennes 1.fr/).

\subsection{Imaging system}

Images were acquired on PIXEL (http://pixel.univ-rennes1.fr/) facility of GIS EUROPIA, (University of Rennes1, France) based on a Leica TCS SP2 confocal scanning head coupled to a DMIRE2 inverted microscope and equipped with an IR $80 \mathrm{MHz}$ femtosecond laser (MAITAI, Spectra Physics). A laser beam tuned at $940 \mathrm{~nm}$ was focalized through a 60X water immersion objective (LUMF1 60XW, NA $=1.1$, Olympus). The pulse width at the sample is typically 250 fs. A motorized rotation stage (PR50CC, Newport) equipped with an achromatic zero-order Quartz- $\mathrm{MgF}_{2}$ half-wave plate was used in order to adjust the mean power of the laser beam at the sample. To avoid photodomage (Recher et al 2011) the incident power never exceed $20 \mathrm{~mW}$ in living tail muscle tissue. However, for fixed and thin muscle tissue (used 
for imunolabeling), 5-10 $\mathrm{mW}$ was used to obtain well-contrasted and stable SHG images. This power always induces photo bleaching of the TPEF signal. SHG signal was collected in a forward direction using 0.90 NA multi-immersion condenser (model 501000, Leica). BG39 bandpass and a $470 \pm 5 \mathrm{~nm}$ interference filters were placed in front of the PMT. For dual SHG and TPEF imaging a laser beam tuned at $860 \mathrm{~nm}$ was used to enable efficient excitation of Alexa Fluor 594 goat antimouse IgG antibody. BG39 bandpass and $430 \pm 5 \mathrm{~nm}$ interference filters were placed in front of the transmission PMT to record the forward SHG signal while BG39 bandpass and 600-700 bandpass filters were placed in front of the backward PMT to record TPEF signal.

\subsection{Image analysis and theoretical simulations}

TPEF and SHG images were processed by a fast Fourier transform filter and were analyzed using open source ImageJ software (http://rsb.info.nih.gov/ij/). As previously reported, the percentage of $2 \mathrm{f}$ sarcomeric SHG-IPs was quantified for each frame using either pixel intensity profile analysis along myofibrils main axis or a FFT analysis followed by a two-band pass Gabor filtering method (Recher et al., 2009).

Quantification of myofibrillar misalignment was undertaken by measuring the average misregistered distance separating adjacent sarcomeric A-bands from random chosen TEM images of both premetamorphic and metamorphic Xenopus laevis tadpoles tail muscles.

Theoretical calculation of SHG and TPEF intensities emitted by a bundle of myofibrils has been done elsewhere (Rouede et al., 2013a; Rouede et al., 2013b; Rouede et al., 2011; Rouede et al., 2014). It is obtained by summation of the amplitude (SHG) or the intensity (TPEF) emitted by all nonlinear sources within the excitation volume (PSF) for each position of the laser beam along the sarcomere. Eq. 3 of Rouede et al. (2014) (Rouede et al., 2014) is used for the calculation of theoretical SHG, Z-band and A-band TPEF intensity patterns.

\section{Results}

Fragmentation of muscle fibers into a number of so-called apoptotic bodies with wellpreserved cross-striations is an histological characteristic change occurring in metamorphosing tail muscles at climax stages 60-64 (Kerr et al., 1974). Typical Z-stack sequence of SHG images taken from live 63 climax stage tadpole tail muscles, within these apoptotic bodies is illustrating in panels A and B of Fig. 1.

Several disorganized fragments of myofibers (dotted line) and well-preserved myofiber (continuous line) can be seen respectively in the ventral and dorsal regions (Fig. 1B). Corresponding sarcomeric SHG-IP are $2 \mathrm{f}$ (ventral) and $1 \mathrm{f}$ (dorsal) as indicated in Fig. 1C. In apoptotic bodies the percentage of $2 \mathrm{f}$ sarcomeric SHG-IP was found to be $97,7 \pm 0,5 \%(\mathrm{n}=64$ images). SHG intensity was very high in apoptotic bodies probably due to an increased condensation of sarcomeric A-bands (see Fig. 2). They are also regions within apoptotic bodies where striation pattern is lost indicating advanced proteolysis (data not shown).

It has been reported at EM level that, following fiber fragmentations, apoptotic bodies of amphibian tail muscle were invaded by macrophages, engulfed into their vacuoles called phagosomes where proteolysis leads to progressive loss of structural organization (Kerr et al., 1974). Since apoptotic bodies are characterized by predominant $2 \mathrm{f}$ sarcomeric SHG-IP due to drastic proteolysis, we undertook EM studies in order to determine the structural origin of such pattern. Typical EM image of apoptotic body from metamorphic climax stage 63 xenopus tail is illustrated in Fig. 2. A membrane-delimited vesicle or vacuole containing several fragments of myofibrils and a cluster of dead mitochondria can be observed inside the cytoplasm of a macrophage. It becomes evident, at higher magnification, and after a 50degree counter-clock rotation of the whole image that fragments of myofibrils are not 
registered at their Z-bands (Fig. 2B). We found that adjacent sarcomeres are misaligned by more than $150 \mathrm{~nm}$ (Fig. 2C).

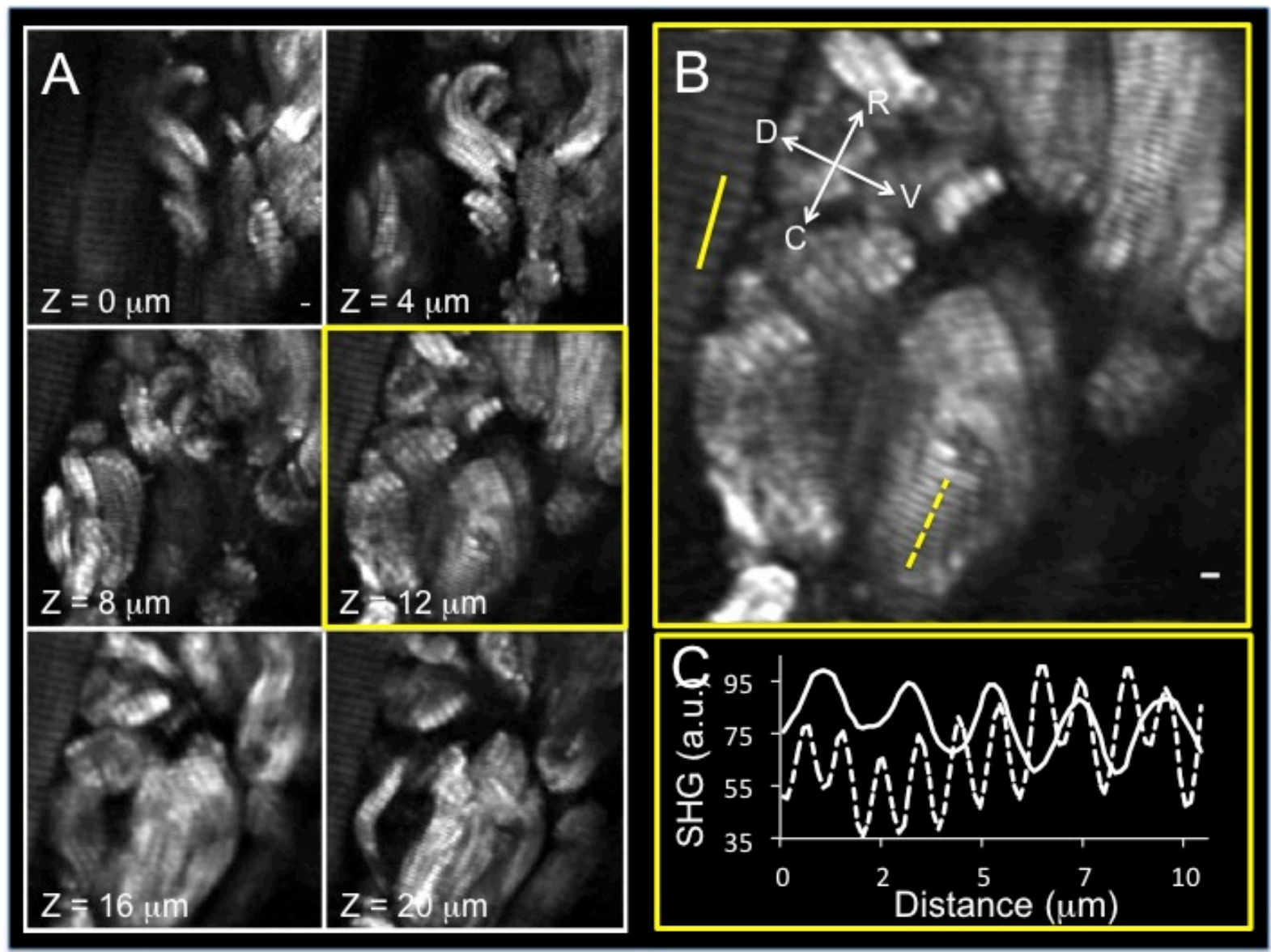

Figure 1: Typical SHG images illustrating fragmentation of muscle fibers occurring at metamorphic climax stage 63 in live xenopus tail muscle. (A) Sequential SHG images from a Z-stack and spaced by $4 \mu \mathrm{m}$ as indicated. The indicated scale bar in the first panel is $2 \mu \mathrm{m}$ and is for all panels. (B) Enlarge view of the image taken at $\mathrm{z}=12 \mu \mathrm{m}$. Continuous and dotted lines indicates portions of respectively well-preserved and fragmented fibers. Scale bar is $2 \mu \mathrm{m}$. (C) Continuous and dotted line curves are SHG intensity profiles in arbitrary units (a.u.) along indicated lines in (B). Note that continuous and dotted line curves correspond respectively to continuous and dotted lines of (B). Note also that sarcomeres of well-preserved and fragmented portions of fibers have respectively 1f and $2 \mathrm{f}$ sarcomeric SHG-IP. Note the indicated orientation of the tail in $\mathrm{B}$ ( $\mathrm{D}=$ dorsal; $\mathrm{V}=$ ventral; $\mathrm{R}=$ rostral; $\mathrm{C}=$ caudal).

We found that the average myofibrillar misalignment was $277 \pm 20 \mathrm{~nm}(\mathrm{n}=28$ images). As a consequence, triads composed of the association between a central T-tubule and two SR cisternae are completely absent. SR is also absent between condensed myofibrils. Altogether EM images obtained from phagosomes show a good correlation between predominant $2 \mathrm{f}$ sarcomeric SHG-IP, myofibrillar misalignment and triads disappearance in agreement with results reported for mdx muscle (Rouede et al., 2014). 


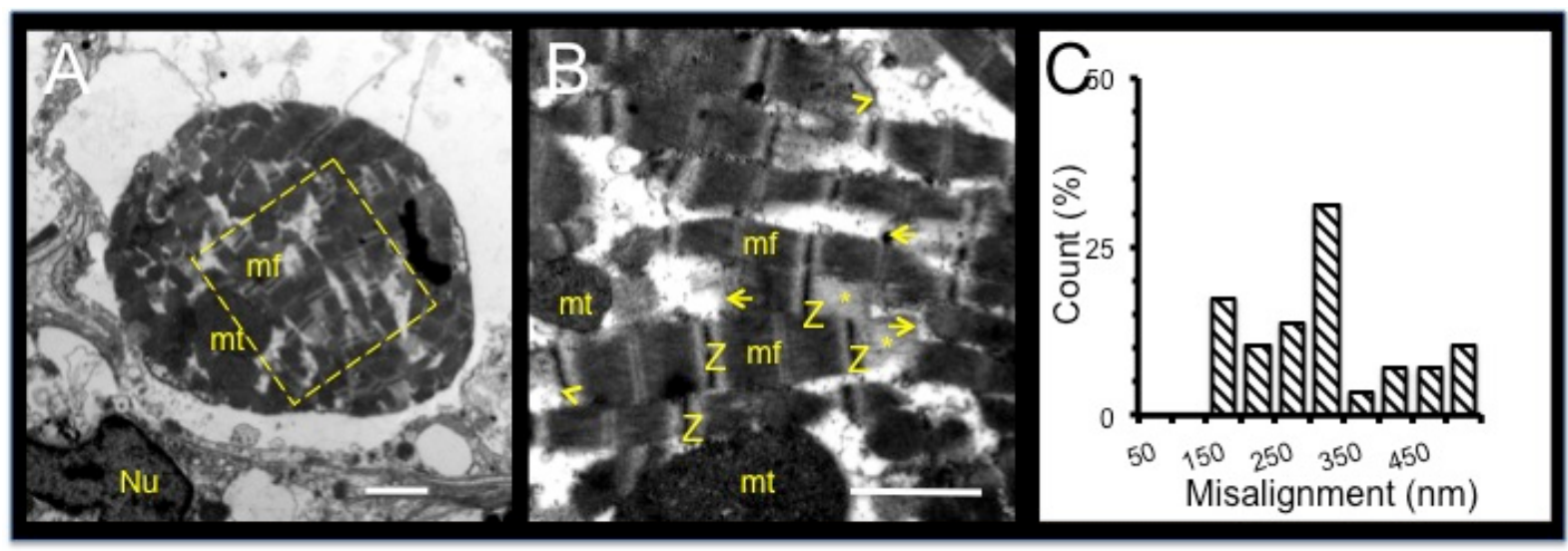

Figure 2: TEM images of metamorphic climax stage 63 tail muscle fragment and quantification of myofibrillar misalignment. (A) Typical TEM images showing a vesicular packed muscle fragments (apoptotic body) inside the cytoplasm of a macrophage. Several fragments of myofibrils (mf) and a cluster of dead mitochondria $(\mathrm{mt})$ can be observed. A Nucleus $(\mathrm{Nu})$ with condensed chromatin can also be seen (lower left corner). (B) Higher magnification of the image region indicated by the rectangular ROI in A. Note the counter clock 50 degrees rotation of the ROI. Note also the longitudinal displacement of myofibrils relative to each other, their condensation (lost of sarcoplasmic reticulum), the presence of half sarcomeres with lost of A-band thick filaments (asterisks), absence of triads (association between two sarcoplasmic reticulum cisternae and a central T-tubule). Note also sarcomere fractured at either I- (arrowhead) or A-bands (arrows). Scale bar are $2 \mu \mathrm{m}$. (C) Histogram representing frequency distribution of misaligned sarcomeres (sub-class increment is $50 \mathrm{~nm}$ ).

It has also been shown that TH induced spatial dilatation of SR is the mechanism that precedes and generates muscle fragmentation and apoptotic bodies (Kerr et al., 1974). To study the effect of local SR dilatation prior muscle fragmentation on the appearance of $2 \mathrm{f}$ sarcomeric SHG-IP, we compare the percentage of this pattern on 3 characteristic different developmental stages. To this aim, we choose stages 50-53 premetamorphic (no TH), stages 55-57 prometamorphic (low level of TH induced hindlimb growth) and metamorphic climax stages 60-63 (high level of TH induced tail resorption) xenopus tadpoles. The results are shown in Fig. 3A. We found that stages 50-53 premetamorphic and stages 55-57 prometamorphic tadpoles non-fragmented muscles were characterized by scarce $2 \mathrm{f}$ sarcomeric SHG-IP (image not shown) in agreement with our previous report (Recher et al., 2011a). Quantifications of the percentage of $2 \mathrm{f}$ sarcomeric SHG-IP in non-fragmented muscles (Fig. 3A) were found to be respectively $7,1 \pm 0,9 \%(\mathrm{n}=214$ images) and 7,3 $\pm 1,4 \%(\mathrm{n}=82$ images) and were not significantly different. However, drastic and significant increase in the proportion of $2 \mathrm{f}$ sarcomeric SHG-IP $(66,1 \pm 3,0 \% ; \mathrm{n}=124$ images) was observed in nonfragmented muscles at metamorphic climax stage 60-63 at time where $\mathrm{TH}$ has been shown to induce drastic increase in $\mathrm{TH}$ receptors (TR) expression in tail muscles (Fujimoto et al., 2007). This latter result is illustrated in Fig. 3B as a 3D orthogonal views from $6 \mu$ m thick Zstack. The XY image (left panel) shows a mixed 1f and $2 \mathrm{f}$ sarcomeric SHG-IP located respectively on top and bottom part of the image. Two orthogonal XZ slices (b1) and (b2) at horizontal lines 1 and 2 in (b0) show that $2 \mathrm{f}$ sarcomeric SHG-IP is partially or fully observed through the $6 \mu \mathrm{m}$ thickness of the stack. Individual myofibrils could not be resolved in the YZ slices (b3) and (b4) corresponding to transverse section through adjacent sarcomeres. Therefore no information concerning SHG-IP modification was available in YZ views. 


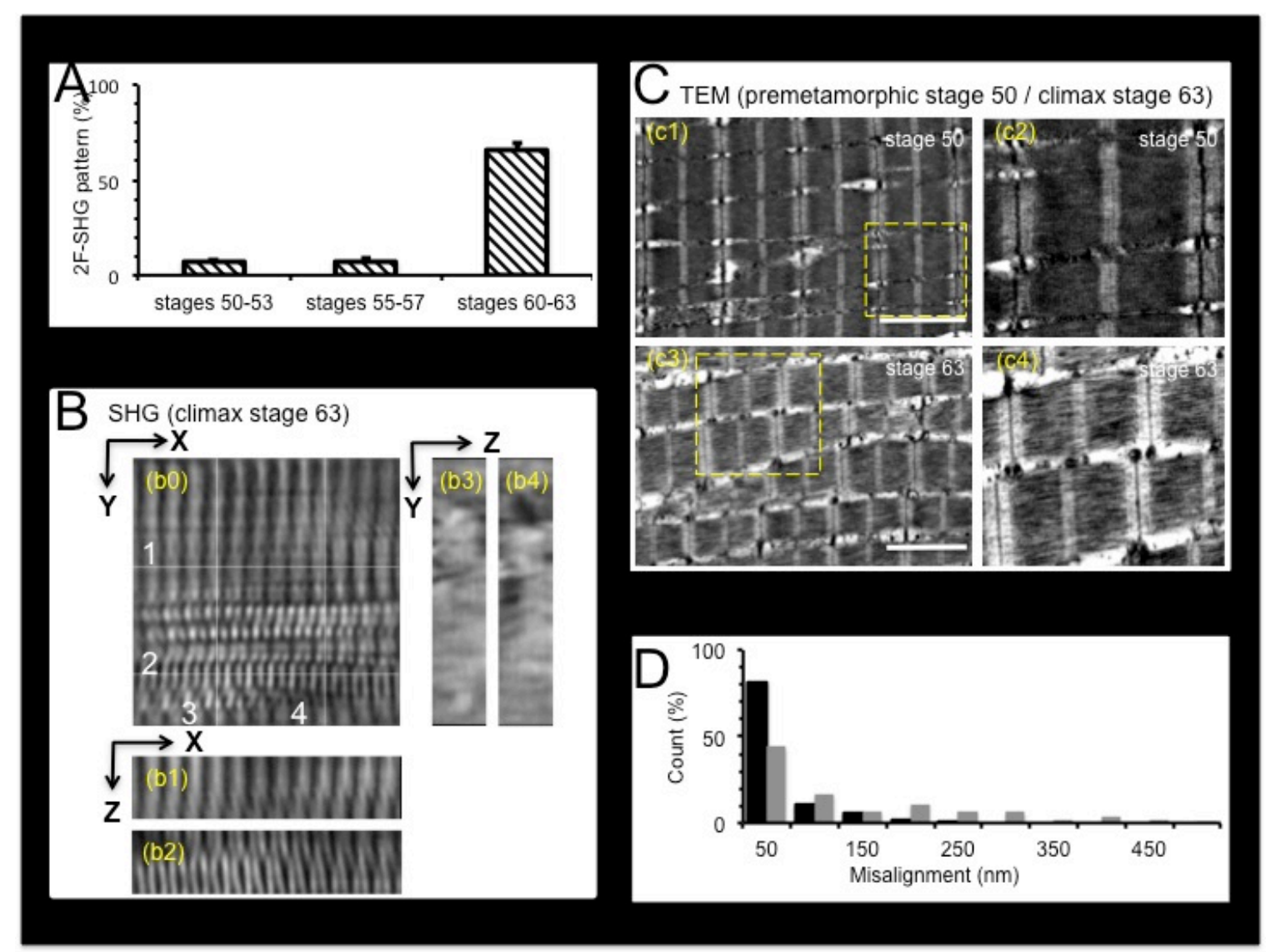

Figure 3: Relationship between SHG and TEM images of non-fragmented tail muscles from different developmental stages xenopus tadpoles. (A) Percentage of 2f sarcomeric SHG-IP of premetamophic (stages 50-53), prometamorphic (stages 55-57) and metamorphic climax (stages 60-63) stages of xenopus tadpoles. (B) 3D orthogonal $\mathrm{XY}, \mathrm{XZ}$ and $\mathrm{YZ}$ views of SHG images from live non-fragmented tail muscle region of metamorphic climax stage 63 tadpole. Panel (b0) represents XY view $(25 \mu \mathrm{m} \times 25 \mu \mathrm{m})$ of the last slice of a Z-stack. Panels (b1) and (b2) are XZ reslice views $(25 \mu \mathrm{m} \times 6 \mu \mathrm{m})$ obtained respectively along horizontal lines 1 and 2 indicated in the XY view (b0). Panels (b3) and (b4) are the YZ reslice views (25 $\mu \mathrm{m} \times 6 \mu \mathrm{m}$ ) obtained respectively along vertical lines 3 and 4 indicated in the XY view (b0). (C) TEM images from tail muscles, at premetamorphic stage 50 (panels (c1), (c2)) and metamorphic climax stage 63 (panels (c3), (c4)). (c2) and (c4) are enlarged images of ROIs indicated in panels (c1) and (c3). Note that sarcomeres are well registered and triads are preserved in panels (c1) and (c2) from premetamorphic tadpole tail muscle. In contrast myofibril are misaligned and severed triads can be observed in panels (c3) and (c4) from metamorphic climax stage 63. Scale bars $=2 \mu \mathrm{m}$. (D) Histogram representing frequency distribution of misaligned sarcomeres from premetamorphic (black bars) and climax stage (grey bars) xenopus tadpoles (sub-class increment is $50 \mathrm{~nm}$ ).

Based on the drastic increase in $2 \mathrm{f}$ sarcomeric SHG-IP in XY and XZ views of SHG images of non-fragmented muscle at climax stage, we were confident that its structural origin could be determined at EM level. To this aim, we compared EM images of non-fragmented muscles from premetamorphic and metamorphic climax stage tadpoles as illustrated in Fig. 3C. Whereas the former exhibit well registered myofibrils with clear visible triads, the latter were characterized by misaligned adjacent sarcomeres with disrupted and severed triads. The frequency of misaligned adjacent sarcomeres was significantly greater $(p<0,001)$ in metamorphic climax compared to premetamorphic stage tadpoles (Fig. 3D). The average misaligned values were found to be respectively $108 \pm 10 \mathrm{~nm}(\mathrm{n}=144$ images $)$ and $21 \pm 3 \mathrm{~nm}$ 
( $\mathrm{n}=194$ images). Interestingly at these metamorphic climax stages the SR exhibits swelling suggesting that this dilatation might be the cue of triad disruptions leading to myofibrillar misalignment and appearance of $2 \mathrm{f}$ sarcomeric SHG-IP.

Altogether these results indicate that $2 \mathrm{f}$ sarcomeric SHG-IP is well correlated to myofibrillar misalignment and triads damage observed at EM level in non-fragmented tail muscles of metamorphic climax stage tadpoles. Moreover $2 \mathrm{f}$ sarcomeric SHG-IP appeared to be a signature of SR swelling that induced triad disruptions and myofibrillar misalignment.

Since disruption of triads resulting in myofibrillar misalignment may have detrimental consequence to excitation-contraction coupling with either muscle weakness or disease, mapping and quantification of label-free $2 \mathrm{f}$ sarcomeric SHG-IPs may emerge as powerful biomedical probe. We next compare sensitivity of SHG over TPEF microscopy to detect myofibrillar misalignments. To this aim we use sarcomeric Z-band $\alpha$-actinin and A-band myosin fluorescent probes.

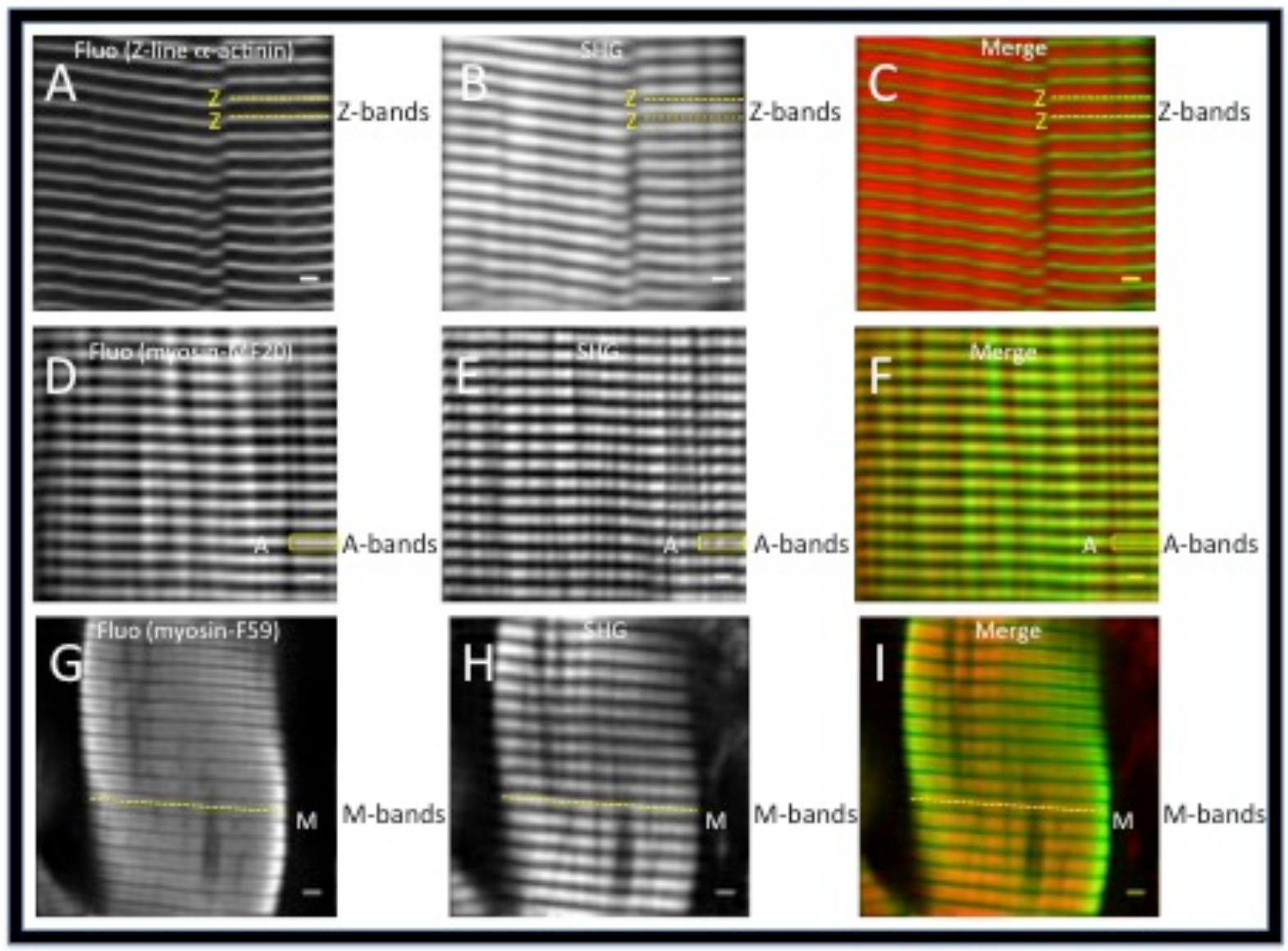

Figure 4: Typical duplex TPEF (A, D, G), SHG (B, E, H) and merge (C, F, I) images of non-metamorphosing tail muscles of xenopus tadpoles. Note that for merge images $(\mathrm{C}, \mathrm{F}, \mathrm{J})$ TPEF is green and SHG is red. (A, B, C) are respectively multiplex $\alpha$-actinin TPEF, SHG and merge images from the same field of view of a stage 57 prometamorphic xenopus tadpole. The dotted segments are ROIs delimiting the same Z-bands of contiguous sarcomeres belonging to different myofibrils that cannot be resolved. (D, E, F) are respectively multiplex myosin TPEF (MF-20 antibody), SHG and merge images from the same field of view of a stage 57 prometamorphic xenopus tadpole. The small rectangle boxes are ROIs indicating the same contiguous A-bands of adjacent myofobrils. Note that MF20 antibody labels the myosin molecules tails and therefore produces a good colocalization of TPEF and SHG signals. (G, H, I) are respectively multiplex myosin TPEF (F59 antibody), SHG and merge images from the same field of view 
of a stage 50 premetamorphic xenopus tadpole. Dotted segments are ROIs indicating the same contiguous M-bands (center of myosin thick filaments lacking cross bridges) of adjacent sarcomeres. Note that F59 antibody labels myosin cross bridges (head of myosin molecules) and therefore no TPEF signal is expected at the M-band. Note also that SHG signal covers whole A-bands. Scale bars are $2 \mu \mathrm{m}$.

For normal tail muscles of stage 57 xenopus tadpoles, we found that TPEF signals from $\alpha$-actinin (Z-band marker) generate banding patterns alternating with that of SHG (originating from A-bands) as illustrated in Fig. 4A-C in agreement with our previous result in normal mouse (Rouede et al., 2014) and xenopus (Recher et al., 2009) gastrocnemius muscles. SHG signals (Fig. 4D-I) were found to fully colocalize with myosin-based TPEF from MF20 antibody targeting myosin tails (Shimizu et al., 1985). A discrepancy was observed between SHG and myosin-TPEF for F59 antibody targeting myosin cross bridges (Miller et al., 1989). In this latter case TPEF signal was reduced at the M-bands (centers of myosin thick filaments voided of myosin cross bridges) as expected from the optical resolution of the microscope $(0.4 \mu \mathrm{m})$. Altogether the localizations of myosin-based TPEF signals are in agreement with their structural origin. For SHG signal a paradoxical bright intensity was observed at the Mband despite the centrosymmetry resulting from antiparallel organization of myosin tail. This over intensity of the SHG signal at the M-band was suggested to originate from particular constructive interferences between harmonic waves (Rouede et al., 2013a; Rouede et al., 2011).

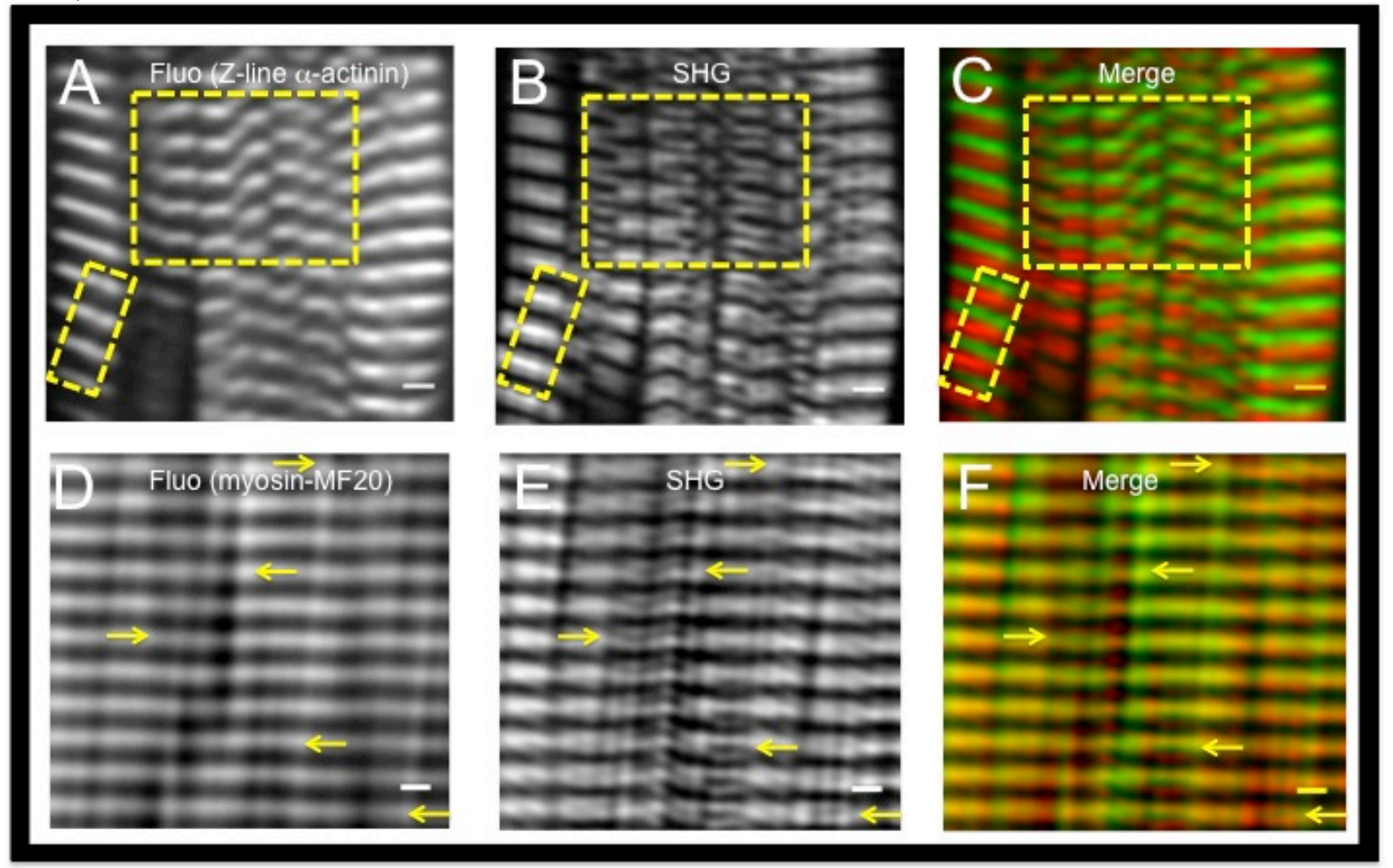

Figure 5: Typical duplex TPEF (A, D), SHG (B, E) and merge (C, F) images from $\alpha$-actinin and myosin of proteolyzed non-fragmented stage 60 xenopus tadpole tail muscles. Note that for merge images (C, F) TPEF is in green and SHG is in red color. (A, B, C) are respectively multiplex $\alpha$-actinin TPEF, SHG and merge images from the same field of view. Large rectangle ROIs represent the same regions where Z-band misalignments and 2f sarcomeric SHG-IP are correlated. Small rectangle ROIs represent the same regions with registered Z-bands and good alternation of $\alpha$-actinin TPEF and SHG signals. (D, E, F) are respectively multiplex myosin TPEF (MF-20 antibody), SHG and merge images from the same field of view. Note that arrows indicate examples of region with $2 \mathrm{f}$ sarcomeric SHG-IP not observed in corresponding TPEF images. Scale bars are $2 \mu \mathrm{m}$. 
For proteolyzed non-fragmented muscle fibers of metamorphic climax stages xenopus tails, several regions of $2 \mathrm{f}$ sarcomeric SHG patterns are correlated to misaligned Z-bands revealed by $\alpha$-actinin-based TPEF signal (see large box ROIs in Fig. 5A-C). We also noticed that $\alpha$-actinin TPEF signals partially reveal regions of $2 \mathrm{f}$ sarcomeric SHG in agreement with our previous report on mdx mouse muscle (Rouede et al., 2014). However, myosin-based TPEF signal originating from myosin tails labeled with MF20 antibody could not reveal regions characterized by $2 \mathrm{f}$ sarcomeric SHG-IP (indicated by arrows in Fig. 5D-F).

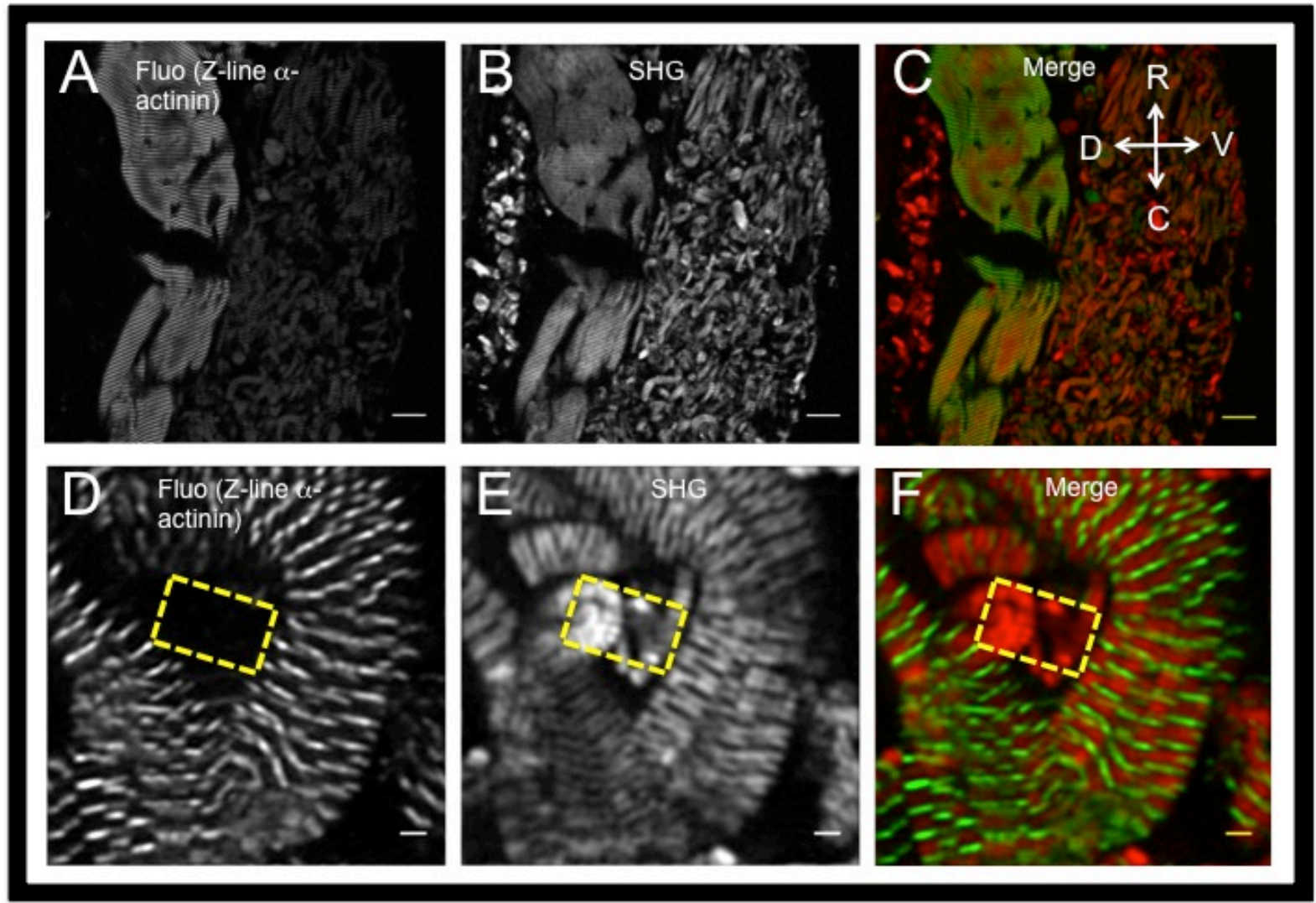

Figure 6: Typical duplex $\alpha$-actinin TPEF (A, D), SHG (B, E) and merge (C, F) images of tail muscle fragments forming apoptotic bodies from climax stage 64 xenopus tadpole. Note that for merge images (C, F) TPEF is in green and SHG is in red color. Note also the indicated orientation of the tail in $\mathrm{C}(\mathrm{D}=$ dorsal; $\mathrm{V}=\mathrm{ventral}$; $\mathrm{R}=$ rostral; $\mathrm{C}=$ caudal) and that proteolyzed dorsal dermal collagen is observed only in SHG image. Note also the presence of dorsal row of slow myofibers that are preserved. (D, E, F) are respectively multiplex $\alpha$-actinin TPEF, SHG and merge images from the same field of view. Note the global correlation between misaligned $\alpha$-actinin TPEF signal and $2 \mathrm{f}$ sarcomeric SHG-IP. Rectangle ROIs represent the same region characterized by disappearance of Z-band $\alpha$-actinin signal suggesting its complete proteolysis whereas sarcomeric A-band SHG signal is still preserved. Scale bars $=2 \mu \mathrm{m}$.

In apoptotic bodies from late climax metamorphic tail muscles (Fig. 6A-C), abundant Zband misalignments revealed by $\alpha$-actinin TPEF signal were often correlated with $2 \mathrm{f}$ sarcomeric SHG-IP (Fig. 6D-F). Moreover, we also found regions with 2 f sarcomeric SHG-IP without $\alpha$-actinin TPEF signal indicating complete proteolysis of sarcomeric Z-band $\alpha$ actinin preceding proteolysis of A-band (see rectangle ROIs in Fig. 6D-E).

Altogether these results demonstrate that SHG-IP is more sensitive than $\alpha$-actinin TPEF signal to report myofibrillar misalignment whereas myosin based TPEF signal is not suitable to report this misalignment. 


\section{Discussion}

In this report, we show that the canonical $1 \mathrm{f}$ sarcomeric SHG-IP of pretamorphic xenopus tadpole tail muscles is converted to $2 \mathrm{f}$ sarcomeric SHG-IP at metamorphic climax stages due to massive physiological muscle proteolysis resulting in myofibrillar misalignment observed with EM. We have also compared for the first time, myosin based TPEF and SHG intensity patterns in normal and metarmophosing tail muscles leading to the conclusion that SHG microscopy is more sensitive than TPEF microscopy to detect and to map sarcomere pattern disruption.

The drastic increase in $2 \mathrm{f}$ sarcomeric SHG-IP occurring at metamorphic climax stages 6063 in tail muscle of xenopus tadpoles coincides with the increase in both TH level and TR expression (Fujimoto et al., 2007; Nakajima et al., 2012) that trigger muscle proteolysis and program cell death. This result is in agreement with our previous report showing that post mortem proteolysis of adult xenopus and rat gastrocnemius muscles results in preponderant $2 \mathrm{f}$ sarcomeric SHG-IP (Recher et al., 2009). Predominance of $2 \mathrm{f}$ sarcomeric SHG-IP in apoptotic bodies of live xenopus tails muscle reported herein was crucial for the ultrastructural determination of their origin at EM level. This has enabled the successful correlation of $2 \mathrm{f}$ sarcomeric SHG-IPs to EM myofibrillar misalignment in agreement with our previous report in mdx muscle (Rouede et al., 2014).

Whereas in human Duchenne muscular dystrophy (DMD) muscles, hyper contraction and stretching of the muscle at different locations were responsible for the breakdown of the intermediate filament protein desmin that connect adjacent sarcomeric Z-band (Cullen et al., 1992), in metamorphosing xenopus tadpole, a different mechanism of myofibrillar misalignment is involved. It has been suggested that muscle fragmentation into apoptotic bodies is due to dilatation and confluence of elements of SR triggered by high levels of TH signaling (Kerr et al., 1974). SR swelling was clearly observed in non-fragmented muscles of xenopus climax tadpoles (Fig. 3) and is sufficient to drive T-tubules disruption and myofibrillar misalignment that we observed both in fragmented and non-fragmented muscles. Altogether the result suggests that the increase in $2 \mathrm{f}$ sarcomeric SHG-IP in non-fragmented muscles $(66 \%)$ might precede their appearance in apoptotic bodies $(97 \%)$ where further amplification of myofibrillar misalignment is generated by macrophage induced phagocytosis and efficient proteolysis. Since the cascade of molecular events linking TH signaling to SR dilatation is not elucidated, $2 \mathrm{f}$ sarcomeric SHG-IP offers a new method to decipher the underlying mechanism. Interestingly, we have previously shown in premetamorphic xenopus tail muscles that $1 \mathrm{f}$ sarcomeric SHG-IP could be converted into $2 \mathrm{f}$ sarcomeric SHG-IP by ROS (Recher et al., 2011a). Also, a good correlation between a chronic increase in concentration of ROS (Tidball and Wehling-Henricks, 2007) and an increase in $2 \mathrm{f}$ sarcomeric SHG-IP (Buttgereit et al., 2013; Friedrich et al., 2010; Garbe et al., 2012; Rouede et al., 2013a; Rouede et al., 2014) can be established in mdx muscular dystrophy. For metamorphic climax stage of xenopus tadpoles, the mechanism of TH induced ROS production is not elucidated. TH can exert direct action on mitochondria uncoupling and generate ROS, but it can also up regulate the expression of NO synthase enzymes leading to NO induce mitochondria ROS production (Inoue et al., 2004; Johnson et al., 2013; Kashiwagi et al., 1999; Menon and Rozman, 2007).

In this study, we have also compared for the first time, myosin based TPEF and SHG intensity patterns in normal and metarmophosing tail muscles. For normal muscle, we found a good correlation between TPEF-IP signals based on primary MF20 monoclonal antibody targeting myosin tails (Shimizu et al., 1985) and 1f SHG-IP (Fig. 4). However, for F59 monoclonal antibody targeting only myosin cross-bridge (Miller et al., 1989) with no signal emitted at the M-band, the expected TPEF intensity patterns were $2 \mathrm{f}$ due to the good optical resolution $(400 \mathrm{~nm})$ of the imaging system. 1f sarcomeric SHG-IP in thick muscle tissue has 
been previously explained to be based on particular coherent property of the diffracted SHG signal taking into account the centrosymmetric properties of the M-band at the middle of each sarcomere (Rouede et al., 2011). For metarmophic climax stages characterized by muscle proteolysis, MF 20 based TPEF-IP remains 1f (Fig. 5) whereas sarcomeric SHG-IP is converted to $2 \mathrm{f}$. In order to understand this discrepancy and to clearly correlate incoherent TPEF and coherent SHG optical signals to myofibrillar misalignment, we undertook theoretical simulation. Theoretical intensity patterns of SHG, MF20- and alpha $\alpha$-actinin based TPEF signals are obtained by calculation (Rouede et al., 2014) of the optical intensity emitted from a bundle of myofibrils centered at $\mathrm{x}=\mathrm{y}=\mathrm{z}=0$ as indicated in the first panel (I) of (Fig. 7). Laser beam is propagating in the $\mathrm{z}$ direction and is focused at the center $(\mathrm{z}=0)$ of the bundle with myofibrillar main axis along the $\mathrm{x}$ direction. For simplicity of the calculation, myofibrillar section is chosen rectangular $(1 \times 1 \mu \mathrm{m})$ (Rouede et al., 2014). We have considered the case of well-aligned myofibrils (Fig. 7a) and of a single misaligned myofibril (Fig. 7b).

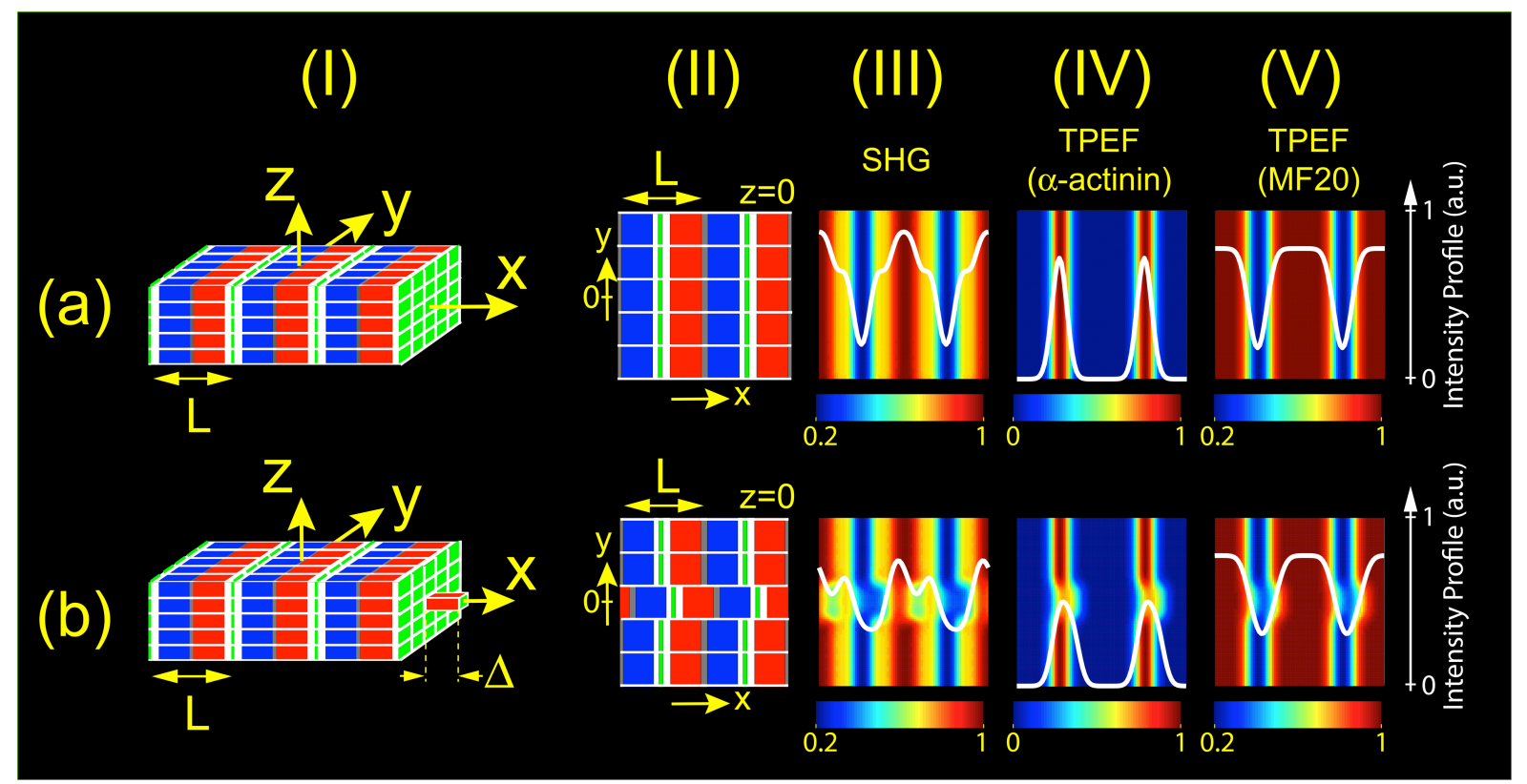

Figure 7: Macroscopic diagrams illustrating the better sensitivity of SHG over TPEF to detect myofibrillar misalignment. (aI) and (bI) are macroscopic xyz view representing respectively well aligned myofibrils and single central myofibril misaligned by $\Delta=0.3 \mu \mathrm{m}$. (aII) and (bII) are corresponding xy views at the center of the bundles $(\mathrm{z}=0)$. For SHG and TPEF simulations, laser beam is propagating along $\mathrm{z}$ direction and is focused at the center of each bundle $(\mathrm{z}=0)$. (aIII) and (bIII) are corresponding SHG patterns. (aIV, aV) and (bIV, bV) are corresponding TPEF patterns from respectively $\alpha$-actinin and myosin tails. Note that macroscopic diagrams (aI, bI) represent bundles of 3 sarcomeres $(\mathrm{L}=2 \mu \mathrm{m})$ from $5 \times 5$ myofibrils of rectangular size $1 \times 1 \mu \mathrm{m}$ in $\mathrm{y}$ and $\mathrm{z}$ directions. Note also that misalignment (bI, bII) only concerns the central myofibril as indicated. For schematic views (aI, bI, aII, bII), A-bands $(1.6 \mu \mathrm{m})$ consist of well-ordered thick filaments that are represented by a double color code (blue and red) to account for polarity inversion at the M-band $(0.15 \mu \mathrm{m}$, grey color) and $\alpha$-actinin Z-bands $(0.1 \mu \mathrm{m})$ are in green color. Note that for each pattern (III, IV, V), intensity profile (white line) calculated at $\mathrm{y}=\mathrm{z}=0$ is shown in inset.

We found a good correlation between experimental (Fig. 4 and Fig. 5) and theoretical simulation (Fig. 7). For well-aligned myofibrils (as indicated in panel (a)) intensity patterns are $1 \mathrm{f}$ for both SHG (III) and TPEF (IV, V) in agreement with our previous report concerning normal gastrocnemius muscle (Rouede et al., 2011). For a single myofibril misaligned by $\Delta=0.3 \mu \mathrm{m}$ as indicated in panel (b), SHG-IP is $2 \mathrm{f}$ (III) and TPEF is $1 \mathrm{f}$ (IV, V). Such 
discrepancy originates from respectively the incoherent and coherent properties of the two signals. Because optical resolution $(0.4 \mu \mathrm{m})$ of our imaging system is greater than the myofibrillar misalignment $(\Delta=0.3 \mu \mathrm{m})$, it is expected that this misalignment cannot be detected with TPEF and therefore the pattern is 1f. Due to its coherent nature, the SHG process is more sensitive to such small displacement (Rouede et al., 2013a; Rouede et al., 2014). Theoretical simulation shows that $2 \mathrm{f}$ sarcomeric SHG-IP can be obtained for $\Delta \geq$ $0.2 \mu \mathrm{m}$. This result clearly demonstrates that myofibrillar displacement below the optical resolution observed with EM is responsible for SHG- and TPEF- IPs of figure 5 highlighting the supra resolution power of SHG microscopy. We can also notice a good correlation between predicted misalignment $(\Delta \geq 0.2 \mu \mathrm{m})$ that induces $2 \mathrm{f}$ sarcomeric SHG-IP and observed average misalignment at EM level $\left(\Delta_{\mathrm{EM}} \geq 0.28 \mu \mathrm{m}\right.$, Fig. 2) in apoptotic bodies. For non-fragmented muscles, the observed average misalignment at EM level $\left(\Delta_{\mathrm{EM}}=0.11 \mu \mathrm{m}\right.$, Fig. 3) is probably under estimated and such discrepancy is probably due to the differential effect of EM process on tissues with different myofibrillar density. Theoretical simulation shows that for $\Delta \geq 0.5 \mu \mathrm{m}$ sarcomeric $\alpha$-actinin based intensity pattern becomes $2 \mathrm{f}$ while MF20 remains $1 f$ due respectively to the discontinuity of adjacent Z-bands and to the lack of discontinuity of adjacent A-bands. This study therefore highlights the power of SHG over TPEF signal to map myofibrillar misalignment. Hence, being label-free, SHG is an intravital high contrast imaging modality that is more sensitive than TPEF in reporting myofibrillar misalignment.

Myofibrillar misalignment described herein in metamorphic climax stage xenopus tail muscles at EM level has been previously reported in human dystrophic and DMD muscles (Cullen et al., 1992) and has been suggested to underlie the enhanced force loss observed after eccentric contractions (Blaauw et al., 2010). In addition to the correlation between $2 \mathrm{f}$ sarcomeric SHG-IP and A-band misalignment, we found a second correlation between $2 \mathrm{f}$ sarcomeric SHG-IP and triad reduction in agreement with our previous study in mdx muscle (Rouede et al., 2014). These latters are composed of a central T-tubule, flanked by two closely apposed sarcoplasmic reticulum terminal cisternae (Porter and Palade, 1957) and are essential for physiological excitation-contraction coupling. In normal muscle, triads and myofibrils are regularly ordered in adult skeletal muscles (Franzini-Armstrong et al., 1988; Page, 1965; Porter and Palade, 1957; Veratti, 1961) and are developmentally regulated (Ezerman and Ishikawa, 1967; Veratti, 1961). Structural intimate relation between the two was first observed at the Z-bands level in amphibian tadpole tail muscles (Porter and Palade, 1957), suggesting that the functional efficiency of triads could be coupled to structural perfect myofibrillar alignment at their A-, M-, I- and Z-bands. We suggest that $2 \mathrm{f}$ sarcomeric SHG-IP generated by A-bands misalignment should reflect structural alteration of triads in adult skeletal muscle fibers. This is confirmed herein by the strong correlation between observed triad alteration at EM level in apoptotic bodies and non-fragmented tail muscles of climax stage xenopus tadpole and the corresponding preponderant $2 \mathrm{f}$ sarcomeric SHG-IPs.

This study confirms and extends our previous report demonstrating that $2 \mathrm{f}$ sarcomeric SHG-IP are ultra-structurally correlated to myofibrillar misalignment in mdx mouse model of human DMD (Rouede et al., 2014). Moreover this study suggests similarities between physiological xenopus tadpole tail muscle resorption and DMD. Like in mdx mouse, $2 \mathrm{f}$ sarcomeric SHG-IP in xenopus tadpoles tail resorption is probably a ROS dependent signature of proteolysis induced by cytosolic TH signaling. Unlike the milder proteolysis and muscle damage of mdx mouse model compared to DMD boys (Partridge, 2013), xenopus tail resorption is characterized by massive muscle proteolysis triggered by hormonal $\mathrm{TH}$ signaling. Therefore xenopus resorption tail could be an alternative organism model aimed at exploring new therapy for DMD. The advantages of xenopus tadpoles over mouse encompasses 1) thousand of embryos per mating 2) external embryonic development 3) 
optical transparency for SHG imaging 4) easy access to bath apply pharmacological agents 5) study of gene function in developmental processes.

\section{Conclusions}

In this report, we show that the canonical single frequency sarcomeric SHG-IP of pretamorphic xenopus tadpole tail muscles is converted to double frequency sarcomeric SHGIP in metamorphic climax stages due to massive muscle proteolysis induced myofibrillar misalignment observed at EM level. Using dual myosin-based SHG and TPEF we demonstrate experimentally, for the first time to our knowledge, the better sensitivity of the coherent SHG over the incoherent TPEF signal to reveal molecular organization in muscle tissue. Theoretical simulation shows that a single myofibril misalignment as small as $200 \mathrm{~nm}$ produces $2 \mathrm{f}$ sarcomeric SHG-IP.

Whereas $2 \mathrm{f}$ sarcomeric SHG-IP is predominantly observed in either pathological or experimental induced muscle proteolysis, we report for the first time that physiological metamorphosis induces predominance of $2 \mathrm{f}$ sarcomeric SHG-IP in xenopus tadpole tail muscle. We also suggest that $2 \mathrm{f}$ sarcomeric SHG-IP, which is correlated to myofibrillar misalignment, can be used as a physiological marker of muscle oxidation, proteolysis and disruption of excitation-contraction coupling.

From this study, we suggest that $2 \mathrm{f}$ sarcomeric SHG-IP could be used as signature of triad defect and disruption of excitation-contraction coupling and that xenopus tadpole tail resorption could be use as an alternative or complementary model of Duchenne muscular dystrophy.

\section{Acknowledgements}

We thank Matthieu Fabrega for his technical help. This work was supported by Région Bretagne, Rennes Métropole, Conseil Général d'Ille-et-Villaine, CRITT Santé Bretagne, CBB Developpement Bretagne, Ministère de l'Enseignement Supérieur et de la Recherche and the European Union Federal Funds FEDER.

\section{References}

Berry, D.L., Schwartzman, R.A., Brown, D.D., 1998. The expression pattern of thyroid hormone response genes in the tadpole tail identifies multiple resorption programs. Developmental biology 203, 12-23.

Blaauw, B., Agatea, L., Toniolo, L., Canato, M., Quarta, M., Dyar, K.A., Danieli-Betto, D., Betto, R., Schiaffino, S., Reggiani, C., 2010. Eccentric contractions lead to myofibrillar dysfunction in muscular dystrophy. J Appl Physiol 108, 105-111.

Boulesteix, T., Beaurepaire, E., Sauviat, M.P., Schanne-Klein, M.C., 2004. Second-harmonic microscopy of unstained living cardiac myocytes: measurements of sarcomere length with 20-nm accuracy. Optics letters 29, 2031-2033.

Buttgereit, A., Weber, C., Garbe, C.S., Friedrich, O., 2013. From chaos to split-ups--SHG microscopy reveals a specific remodelling mechanism in ageing dystrophic muscle. The Journal of pathology 229, 477-485.

Campagnola, P.J., Millard, A.C., Terasaki, M., Hoppe, P.E., Malone, C.J., Mohler, W.A., 2002. Three-dimensional high-resolution second-harmonic generation imaging of endogenous structural proteins in biological tissues. Biophysical journal 82, 493-508.

Cullen, M.J., Fulthorpe, J.J., Harris, J.B., 1992. The distribution of desmin and titin in normal and dystrophic human muscle. Acta neuropathologica 83, 158-169.

Du Pasquier, D., Rincheval, V., Sinzelle, L., Chesneau, A., Ballagny, C., Sachs, L.M., Demeneix, B., Mazabraud, A., 2006. Developmental cell death during Xenopus 
metamorphosis involves BID cleavage and caspase 2 and 8 activation. Dev Dyn 235, 2083-2094.

Endo, M., 1964. Entry of a Dye into the Sarcotubular System of Muscle. Nature 202, 11151116.

Ezerman, E.B., Ishikawa, H., 1967. Differentiation of the sarcoplasmic reticulum and T system in developing chick skeletal muscle in vitro. The Journal of cell biology 35 , 405-420.

Franzini-Armstrong, C., Ferguson, D.G., Champ, C., 1988. Discrimination between fast- and slow-twitch fibres of guinea pig skeletal muscle using the relative surface density of junctional transverse tubule membrane. Journal of muscle research and cell motility 9 , 403-414.

Friedrich, O., Both, M., Weber, C., Schurmann, S., Teichmann, M.D., von Wegner, F., Fink, R.H., Vogel, M., Chamberlain, J.S., Garbe, C., 2010. Microarchitecture is severely compromised but motor protein function is preserved in dystrophic mdx skeletal muscle. Biophysical journal 98, 606-616.

Fujimoto, K., Nakajima, K., Yaoita, Y., 2007. Expression of matrix metalloproteinase genes in regressing or remodeling organs during amphibian metamorphosis. Development, growth \& differentiation 49, 131-143.

Garbe, C.S., Buttgereit, A., Schurmann, S., Friedrich, O., 2012. Automated multiscale morphometry of muscle disease from second harmonic generation microscopy using tensor-based image processing. IEEE transactions on bio-medical engineering 59, 3944.

Garcia-Canadilla, P., Gonzalez-Tendero, A., Iruretagoyena, I., Crispi, F., Torre, I., AmatRoldan, I., Bijnens, B.H., Gratacos, E., 2014. Automated cardiac sarcomere analysis from second harmonic generation images. Journal of biomedical optics 19, 056010.

Inoue, M., Sato, E.F., Nishikawa, M., Hiramoto, K., Kashiwagi, A., Utsumi, K., 2004. Free radical theory of apoptosis and metamorphosis. Redox Rep 9, 237-247.

Ishizuya-Oka, A., 2011. Amphibian organ remodeling during metamorphosis: insight into thyroid hormone-induced apoptosis. Development, growth \& differentiation 53, 202212.

Jayasinghe, I.D., Launikonis, B.S., 2013. Three-dimensional reconstruction and analysis of the tubular system of vertebrate skeletal muscle. Journal of cell science 126, 40484058 .

Johnson, J., Manzo, W., Gardner, E., Menon, J., 2013. Reactive oxygen species and antioxidant defenses in tail of tadpoles, Xenopus laevis. Comp Biochem Physiol C Toxicol Pharmacol 158, 101-108.

Kashiwagi, A., Hanada, H., Yabuki, M., Kanno, T., Ishisaka, R., Sasaki, J., Inoue, M., Utsumi, K., 1999. Thyroxine enhancement and the role of reactive oxygen species in tadpole tail apoptosis. Free radical biology \& medicine 26, 1001-1009.

Kerr, J.F., Wyllie, A.H., Currie, A.R., 1972. Apoptosis: a basic biological phenomenon with wide-ranging implications in tissue kinetics. British journal of cancer 26, 239-257.

Kerr, J.F., Harmon, B., Searle, J., 1974. An electron-microscope study of cell deletion in the anuran tadpole tail during spontaneous metamorphosis with special reference to apoptosis of striated muscle fibers. Journal of cell science 14, 571-585.

Launikonis, B.S., Stephenson, D.G., 2002. Tubular system volume changes in twitch fibres from toad and rat skeletal muscle assessed by confocal microscopy. The Journal of physiology 538, 607-618.

Llewellyn, M.E., Barretto, R.P., Delp, S.L., Schnitzer, M.J., 2008. Minimally invasive highspeed imaging of sarcomere contractile dynamics in mice and humans. Nature 454, 784-788. 
Menon, J., Rozman, R., 2007. Oxidative stress, tissue remodeling and regression during amphibian metamorphosis. Comp Biochem Physiol C Toxicol Pharmacol 145, 625631.

Miller, J.B., Teal, S.B., Stockdale, F.E., 1989. Evolutionarily conserved sequences of striated muscle myosin heavy chain isoforms. Epitope mapping by cDNA expression. The Journal of biological chemistry 264, 13122-13130.

Nakajima, K., Fujimoto, K., Yaoita, Y., 2012. Regulation of thyroid hormone sensitivity by differential expression of the thyroid hormone receptor during Xenopus metamorphosis. Genes Cells 17, 645-659.

Nucciotti, V., Stringari, C., Sacconi, L., Vanzi, F., Fusi, L., Linari, M., Piazzesi, G., Lombardi, V., Pavone, F.S., 2010. Probing myosin structural conformation in vivo by second-harmonic generation microscopy. Proceedings of the National Academy of Sciences of the United States of America 107, 7763-7768.

Page, S.G., 1965. A comparison of the fine structures of frog slow and twitch muscle fibers. The Journal of cell biology 26, 477-497.

Partridge, T.A., 2013. The mdx mouse model as a surrogate for Duchenne muscular dystrophy. The FEBS journal 280, 4177-4186.

Plotnikov, S.V., Millard, A.C., Campagnola, P.J., Mohler, W.A., 2006. Characterization of the myosin-based source for second-harmonic generation from muscle sarcomeres. Biophysical journal 90, 693-703.

Plotnikov, S.V., Kenny, A.M., Walsh, S.J., Zubrowski, B., Joseph, C., Scranton, V.L., Kuchel, G.A., Dauser, D., Xu, M., Pilbeam, C.C., Adams, D.J., Dougherty, R.P., Campagnola, P.J., Mohler, W.A., 2008. Measurement of muscle disease by quantitative second-harmonic generation imaging. Journal of biomedical optics 13, 044018.

Porter, K.R., Palade, G.E., 1957. Studies on the endoplasmic reticulum. III. Its form and distribution in striated muscle cells. The Journal of biophysical and biochemical cytology 3, 269-300.

Prent, N., Green, C., Greenhalgh, C., Cisek, R., Major, A., Stewart, B., Barzda, V., 2008. Intermyofilament dynamics of myocytes revealed by second harmonic generation microscopy. Journal of biomedical optics 13, 041318.

Ralston, E., Swaim, B., Czapiga, M., Hwu, W.L., Chien, Y.H., Pittis, M.G., Bembi, B., Schwartz, O., Plotz, P., Raben, N., 2008. Detection and imaging of non-contractile inclusions and sarcomeric anomalies in skeletal muscle by second harmonic generation combined with two-photon excited fluorescence. Journal of structural biology 162, 500-508.

Recher, G., Rouède, D., Schaub, E., Tiaho, F., 2011a. Skeletal muscle sarcomeric SHG patterns photo-conversion by femtosecond infrared laser. Biomed. Opt. Express 2, 374-384.

Recher, G., Rouede, D., Tascon, C., D'Amico, L.A., Tiaho, F., 2011b. Double-band sarcomeric SHG pattern induced by adult skeletal muscles alteration during myofibrils preparation. Journal of microscopy 241, 207-211.

Recher, G., Rouede, D., Richard, P., Simon, A., Bellanger, J.J., Tiaho, F., 2009. Three distinct sarcomeric patterns of skeletal muscle revealed by SHG and TPEF microscopy. Optics express 17, 19763-19777.

Rouede, D., Bellanger, J.J., Recher, G., Tiaho, F., 2013a. Study of the effect of myofibrillar misalignment on the sarcomeric SHG intensity pattern. Optics express 21, 1140411414 
Rouede, D., Bellanger, J.J., Schaub, E., Recher, G., Tiaho, F., 2013b. Theoretical and Experimental SHG Angular Intensity Patterns from Healthy and Proteolysed Muscles. Biophysical journal 104, 1959-1968.

Rouede, D., Recher, G., Bellanger, J.J., Lavault, M.T., Schaub, E., Tiaho, F., 2011. Modeling of supramolecular centrosymmetry effect on sarcomeric SHG intensity pattern of skeletal muscles. Biophysical journal 101, 494-503.

Rouede, D., Coumailleau, P., Schaub, E., Bellanger, J.J., Blanchard-Desce, M., Tiaho, F., 2014. Myofibrillar misalignment correlated to triad disappearance of $\mathrm{mdx}$ mouse gastrocnemius muscle probed by SHG microscopy. Biomed Opt Express 5, 858-875.

Rowe, I., Le Blay, K., Du Pasquier, D., Palmier, K., Levi, G., Demeneix, B., Coen, L., 2005. Apoptosis of tail muscle during amphibian metamorphosis involves a caspase 9dependent mechanism. Dev Dyn 233, 76-87.

Shimizu, T., Dennis, J.E., Masaki, T., Fischman, D.A., 1985. Axial arrangement of the myosin rod in vertebrate thick filaments: immunoelectron microscopy with a monoclonal antibody to light meromyosin. The Journal of cell biology 101, 11151123.

Soeller, C., Cannell, M.B., 1999. Examination of the transverse tubular system in living cardiac rat myocytes by 2-photon microscopy and digital image-processing techniques. Circulation research 84, 266-275.

Takekura, H., Fujinami, N., Nishizawa, T., Ogasawara, H., Kasuga, N., 2001. Eccentric exercise-induced morphological changes in the membrane systems involved in excitation-contraction coupling in rat skeletal muscle. The Journal of physiology 533, 571-583.

Thornberry, N.A., 1997. The caspase family of cysteine proteases. British medical bulletin 53, 478-490.

Tidball, J.G., Wehling-Henricks, M., 2007. The role of free radicals in the pathophysiology of muscular dystrophy. J Appl Physiol 102, 1677-1686.

Veratti, E., 1961. Investigations on the fine structure of striated muscle fiber read before the Reale Istituto Lombardo, 13 March 1902. The Journal of biophysical and biochemical cytology 10(4)Suppl, 1-59.

Yeung, E.W., Balnave, C.D., Ballard, H.J., Bourreau, J.P., Allen, D.G., 2002. Development of T-tubular vacuoles in eccentrically damaged mouse muscle fibres. The Journal of physiology 540, 581-592. 